\title{
Ignition of liquid droplets fuels under conditions of radiation-conductive heating in air
}

\author{
Arkadiy V. Zakharevich ${ }^{1, *}$, Mikhail S. Zygin ${ }^{1}$, and Dmitriy N. Tsymbalov ${ }^{1}$ \\ ${ }^{1}$ National Research Tomsk Polytechnic University, 634050 Tomsk, Russia
}

\begin{abstract}
The results of an experimental investigation of ignition liquid fuel (kerosene, diesel fuel) by the single drops under conditions of radiation-conducting heating in air are presented. The dependences of the ignition delay time of the typical fuels droplets on the initial temperature of the heated oxidant in a limited space are established. The parameters of stable ignition of liquid fuel droplets are set.
\end{abstract}

\section{Introduction}

Combustion of sprayed liquid fuels occurs in many energy systems [1,2], installations $[3,4]$ and apparatus [5,6]. The regularities of the droplet combustion of such fuels have been studied for a long time [7,8] and intensively [9]. However, interest in the combustion of liquid droplets is not reduced to the present time $[10,11]$ due to the complex mechanisms of processes of heat and mass transfer and physicochemical transformations occurring in the drop, on its surface and in the near-surface (or adjacent) layer of the vapor-gas mixture. In particular, up to the present time, the temperature field of droplets is often assumed to be uniform when analyzing the conditions and characteristics of the combustion processes of condensed substances [12]. The rate of vaporization of a flammable liquid is generally assumed to depend only on the intensity of the vapor mass transfer process in a thin layer of the external environment adjacent to the surface of the droplet [11]. At the same time the experimental data on the conditions and characteristics of the processes of ignition of fuel droplets and flammable liquids in the medium of high-temperature gases published relatively little. From the review [11] it can be concluded that there are no mathematical models describing the processes of ignition of condensed substance droplets in the oxidizer medium, as well as in the real possible ranges of changes in the main significant factors (ambient temperature, heat exchange intensity, droplet configuration and conditions of its movement, thermal properties of the flammable liquid, etc.). The base in the description of the combustion of drops of liquid is the model of Warsaw formulated many years ago. But the approach [13] does not provide the possibility to analyze the characteristics of the fuel droplet ignition process (primarily the ignition delay time) depending on the conditions of the thermal effect of the environment.

\footnotetext{
* Corresponding author: bet@,tpu.ru
} 
The aim of the work is to establish the conditions and characteristics of typical liquid fuel droplets ignition under conditions of conductive-radiation heating as a result of experimental studies.

\section{Experimental method}

A specialized experimental stand has been developed to study the integral characteristics of the liquid fuel droplet ignition process (Fig.1), which includes a combustion chamber (1), a high - speed video camera (2) with the "Tema Automotive" software, a motorized coordinate device (3) for moving a drop of fuel at a speed of $0.5 \mathrm{~m} / \mathrm{s}$, an electronic dispenser (4) "Finn pipette" (minimum and maximum volumes to be taken is 1 and $10 \mu 1$ with a variation step of $0.1 \mu \mathrm{l}$ ) for generating drops in the range of 3-8 $\mu 1$ and PC (5).

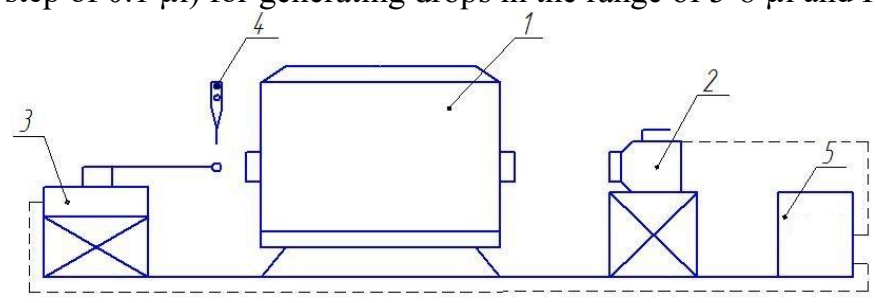

Fig. 1. Schematic diagram of the experimental setup: 1 - tubular oven "Nabertherm R 50/250/13" with integrated temperature controller, 2 - high-speed video camera "Phantom V411", 3 - motorized coordinate device, 4 - electronic dispenser "Finn pipette", 5 - PC.

The objects of the study were widely used liquid fuels - kerosene of the TS-1 brand (GOST 10227-86 with changes 1-3) and winter diesel fuel DT-Z-K3-38 (GOST R 554752013). The experiments were carried out under the same and well reproducible conditions (temperature 290-293 K, relative humidity 50-70\%, atmospheric pressure 730-760 mmHg.V.). A drop of liquid fuel of a certain volume was dropped by the tip of the Finn pipette dispenser onto the thermocouple junction with a diameter of $0.5 \mathrm{~mm}$. At a fixed speed of $0.5 \mathrm{~m} / \mathrm{s}$ a drop of liquid fuel was moved by a coordinate device to the center of the combustion chamber. In order to preserve the integrity of the droplet and minimize the time of its movement in the chamber, the optimal speed of movement of the coordinate device was established. The values of the oxidizer temperature in the combustion chamber varied in the range of $663-873 \mathrm{~K}$ with a systematic error of $\pm 2 \mathrm{~K}$. The temperature range is due to the need to establish the minimum values at which the stable ignition of a drop of liquid fuel was provided. The processes taking place in the vicinity of the distillate fuel droplet were recorded by a color high-speed camera. After processing the video footage of the experiments, the ignition delay times were determined $\left(\tau_{\text {ign }}\right)$. For each type and volume of the droplet at a constant initial temperature $\left(T_{p}\right)$ in the combustion chamber, a series of $(6-$ 8 ) experiments were carried out. The $\tau_{\text {ign }}$ parameter characterized the time from the moment of introduction of a drop of distillate fuel into the tube space of the combustion chamber until the flame appears. After this was determined the average for each constant value of $T_{p}$ the values of $\tau_{\text {ign }}$ and the standard deviation. As a result of the analysis of ignition delay times for the performed experiments, it was found that the coefficients of variation varied from $0.15 \mathrm{sec}$ (at high $\mathrm{T}_{\mathrm{p}}$ ) to $1.1 \mathrm{sec}$ (at relatively low $\mathrm{T}_{\mathrm{p}}$ ).

\section{Results and discussion}

Fig. 2 shows the experimental dependences of the ignition delay time of distillate fuel droplets on the initial temperature in the combustion chamber. The parameters of stable 
ignition of liquid fuel droplets are set. Each fuel is characterized by a minimum value of the ignition process and stable combustion $\left(\mathrm{T}_{\mathrm{p}}\right)$. According to the results of the experiments, it can be concluded that the droplet size has little effect on the ignition delay time of distillate fuels.

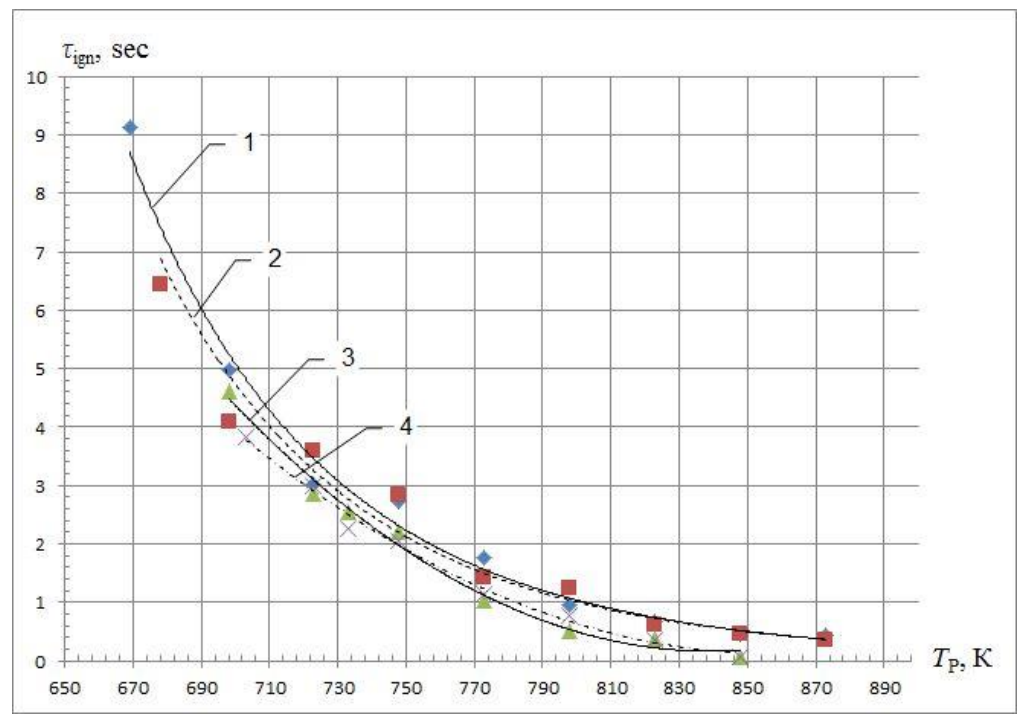

Fig. 2. Experimental dependences of delay time of ignition the drops of distillate fuels from the initial temperature in the combustion chamber: 1,2 -diesel; 3,4-kerosene; 1,3 -drop volume of $7 \mu 1 ; 2,4-$ drop volume of $4 \mu 1$.

Deviations for drops by $\tau_{\text {ign }}$ of $7 \mathrm{ml}$ and $4 \mu 1$ do not exceed random measurement errors of the main characteristics of the process. The mechanism of the established regularities should be investigated in the future using the means of registration of a higher level of detail.

\section{Conclusions}

On the basis of the results of experimental studies, the limiting modes of droplet ignition of fairly typical liquid fuels under conditions of radiation-conductive heating in the air are established. The parameters of stable ignition of liquid fuel droplets are set. The obtained experimental data allow us to conclude that the droplet sizes have little effect on the ignition delay time of distillate fuels.

This work was supported by the Russian Foundation for Basic Research (project No. 17-29-05093).

\section{References}

1. S. Y. Misyura, Nature: Scientific Reports 7, 14759 (2017)

2. S. Y. Misyura, Int. J. Heat Mass Transfer 166, 667 (2018)

3. O. P. Korobeinichev, A. G. Shmakov, V. M. Shvartsberg, A. A. Chernov, S. A. Yakimov, K. P. Koutsenogii, V. I. Makarov, Fire Safety J. 51, 102 (2012)

4. A. Yu. Varaksin, High Temp. 51, 377 (2013)

5. D. Tarlet, C. Allouis, J. Bellettre, Int. J. Therm. Sci. 107, 179 (2017)

6. B. Kichatov, A. Korshunov, A. Kiverin, E. Son, Fuel Proces. Technol. 166, 77 (2017) 
7. E. A. Salgansky, A. Y. Zaichenko, D. N. Podlesniy, M. V. Salganskaya, M. Toledo, Int. J. Hydrogen Energ. 42, 11017 (2017)

8. V. E. Zhukov, A. N. Pavlenko, M. I. Moiseev, D. V. Kuznetsov, High Temp. 55, 79 (2017)

9. S. S. Sazhin, E. A. Shchepakina, V. A. Sobolev, Combust. Flame 187, 122 (2018)

10. K. Warncke, S. Gepperth, B. Sauer, A. Sadiki, J. Janicka, R. Koch, H.-J. Bauer, Int. J. Multiph. Flow 91, 208 (2017)

11. S. S. Sazhin, Fuel 196, 69 (2017)

12. S. S. Sazhin, Prog. Energy Combust. Sci. 2, 162 (2006)

13. G. A. Varshavsky, Proceedings of NII-1 6 (1945) 